report, seizures occurred in 50\% of cases (See Progress in Pediatric Neurology I, PNB Publ, 1991;p318).

\title{
ABSENT LABIAL FRENULUM IN HOLOPROSENCEPHALY
}

Absence of the superior labial frenulum is reported in $88 \%$ of 17 consecutive cases of holoprosencephaly examined at St Christopher's Hospital for Children, Philadelphia, PA. Brain imaging is recommended in children with this anomaly. Patients with midline facial abnormalities such as hypotelorism, smooth philtrum, or single central incisor should be examined for absent frenulum. (Martin RA, Jones KL. Absence of the superior labial frenulum in holoprosencephaly: a new diagnostic sign. I Pediatr July 1998;133:151-153). (Reprints: Rick Martin MD, Section of Clinical Genetics, St Christopher's Hospital for Children, Erie@ Front St, Philadelphia, PA 19134).

COMMENT. Absence of the superior labial frenulum may be added to the spectrum of midline anomalies characteristic of the holoprosencephaly sequence.

\section{TRAUMATIC DISORDERS}

\section{INFLICTED AND NONINFLICTED TRAUMATIC BRAIN INJURY}

Neuroimaging, physical, and neurobehavioral findings after inflicted and noninflicted traumatic brain injury (TBI) in 40 children, 0 to 6 years of age, were compared in a prospective longitudinal study at the University of Texas at Houston Health Science Center, and Baylor College of Medicine, Houston, TX. Signs of preexisting brain injury (cerebral atrophy, subdural hygroma, and ex vacuo ventriculomegaly) were present in $45 \%$ of 20 children with inflicted TBI and in none of 20 with noninflicted TBI. Neuroimaging findings included subdural hematoma in $16(80 \%)$ inflicted TBI cases and $9(45 \%)$ noninflicted cases, and epidural hematoma in none of the inflicted cases and $4(20 \%)$ of noninflicted TBI. Seizures (13 cases) and retinal hemorrhage (13) were significantly more frequent in the inflicted TBI cases than in noninflicted cases: $65 \%$ and $65 \%$ of $15 \%$ and 0 cases, respectively. Skull fractures, cephalhematomas, edema, and intraparenchymal hemorrhage were similar in the two groups. Glasgow Outcome Scale scores, cognitive development, and motor functioning assessed 1.3 months after TBI showed a significantly less favorable outcome after inflicted than noninflicted TBI. Despite comparable Glasgow Coma Scale scores and similar duration of impaired consciousness, the inflicted TBI group had significantly more cases of mental deficiency than the noninflicted TBI group. (Ewing-Cobbs L, Kramer L, Prasad M et al. Neuroimaging, physical, and developmental findings after inflicted and noninflicted traumatic brain injury in young children. Pediatrics August 1998;102:300-307). (Reprints: Dr Linda Ewing-Cobbs, Department of Pediatrics, University of Texas at Houston Health Science Center, 6431 Fannin, Houston, TX 77030).

COMMENT. Features of inflicted traumatic brain injury include acute CT/MRI evidence of old brain injury, subdural hematoma, seizures, retinal hemorrhages, and impaired cognitive function without prolonged impairment of consciousness. Chronic changes on acute CT/MRI in $45 \%$ of children with inflicted TBI, suggesting previous assault and cumulative brain injury, are found without previous reported history of brain injury. Neuroimaging is essential in diagnosis of acute brain injury and for identification of old injuries, especially in shaking-impact injuries which may have no external signs of physical abuse. 